\title{
Superior vena cava syndrome presenting as bilateral breast enlargement with "peau d'orange" skin changes
}

\author{
Gabriela Maloney, J ane J ames, Burnetta Herron, Marylee Braniecki \\ Midwestern University, Chicago, United States.
}

Correspondence: Gabriela Maloney. Address: Midwestern University, Chicago, United States.

Email: gabrielamaloney@gmail.com

Received: November 4, 2014

DOI : $10.5430 /$ crim.v2n2p36

Accepted: November 24, 2014 Online Published: March 11, 2015

URL: http://dx.doi.org/10.5430/crim.v2n2p36

\section{Abstract}

A 51 year old female with significant medical history of end stage renal failure presented with bilateral tender breast enlargement, "peau d'orange" skin changes and bilateral axillary lymphadenopathy. The patient was initially diagnosed with mastitis, and underwent a course of antibiotics with minimal improvement. Extensive imaging and repeated biopsies demonstrated benign breast tissue without malignancy. Punch biopsies of breast skin raised a broad differential diagnosis including neoplasm, fibrosing mediastinitis and superior vena cava obstruction. Immediately following the pathology report which included this differential, a superior vena cava (SVC) occlusion with venous stenotic impairment of lateral cephalic outflow was detected. The patient was then treated with resolution of her symptoms. In this case, SVA occlusion was raised based on the patient's history of chronic renal dialysis requiring multiple catheter placements and by increasing bilateral tender breast enlargement, "peau d'orange" skin changes and histology of lymphedema. We herein describe an unusual case in which the dermatopathologist made the initial awareness of SVA occlusion by seeing lymphangiectasia and stromal edema on a patient with bilateral breast enlargement.

\section{Keywords}

Superior Vena Cava Syndrome, Peau d’orange, End stage renal disease, Chronic hemodialysis, Lymphangiectasia

\section{I ntroduction}

We present a unique case in which skin biopsy findings and the limited history of "peau d'orange" appearance of the breasts led to the detection of a superior vena cava (SVC) occlusion.

\section{Case presentation}

A 51 year old female with significant medical history of end stage renal disease (ESRD) and diabetes mellitus presented with progressive, bilateral, tender breast enlargement, "peau d'orange" skin changes and bilateral axillary lymphadenopathy that had been present for months. The patient also complained of hoarseness, worsening neck enlargement and occasional dysphagia. She was initially diagnosed with mastitis and underwent a course of antibiotics with minimal improvement. Mammograms and breast ultrasonograms demonstrated benign tissue with scattered calcifications and 
cutaneous thickening. Breast core biopsies were benign. CT scan of the abdomen and chest showed anasarca with diffuse edematous changes of the breasts bilaterally, axillary adenopathy, mild pericardial effusion, thyromegaly, and bilateral renal atrophy compatible with the patient's ESRD.

Skin punch biopsies from both breasts demonstrated lymphangiectasia associated with stromal edema, scattered stromal fibroblasts and patchy perivascular plasma cell infiltrates (see Figure 1 and 2). In view of the clinical presentation, the histopathology raised a broad differential diagnosis including occult neoplasm, fibrosing mediastinitis and superior vena cava obstruction. Immediately following release of the pathology report, which included this differential diagnosis, a superior vena cava (SVC) occlusion with venous stenotic impairment of lateral cephalic outflow was detected (see Figure 3). The patient was then treated with $10 \mathrm{~mm}$ and $14 \mathrm{~mm}$ angioplasty balloons, embolization and surgical ligation of retrograde cephalic branches with resolution of her cutaneous symptoms.

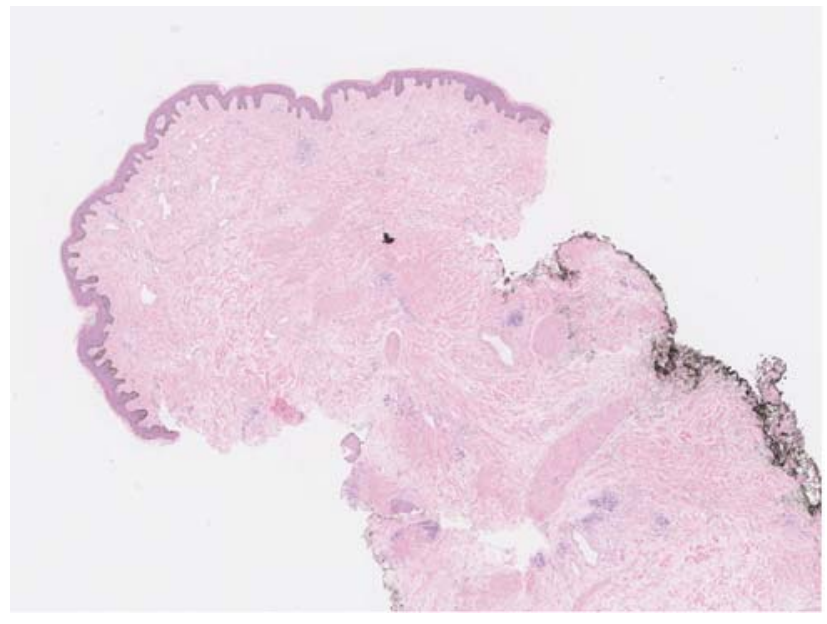

Figure 1. Skin of breast punch biopsy, $(4 \times)$ showing superficial ymphangiectasia.in upper dermis.

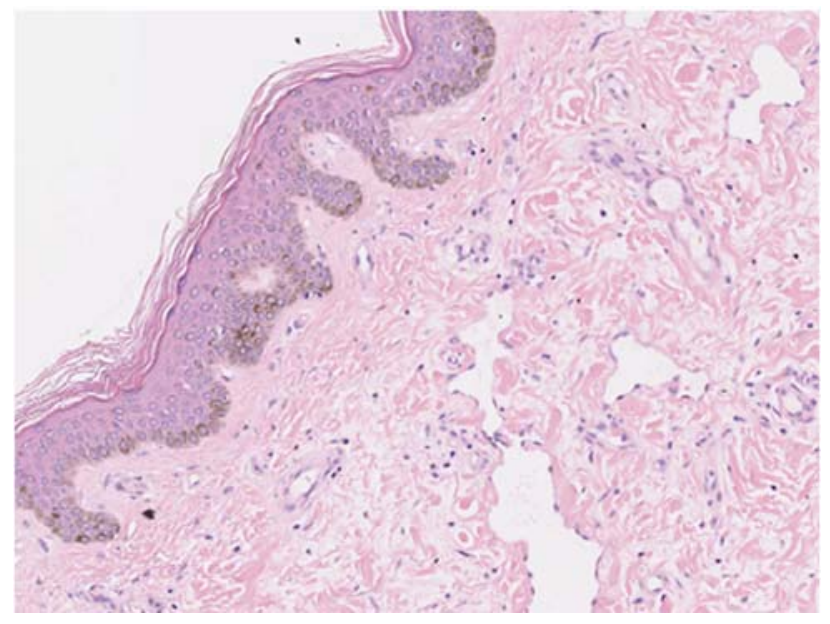

Figure 2. Breast punch biopsy, $(20 \times)$ Dilated lymphatic channels.

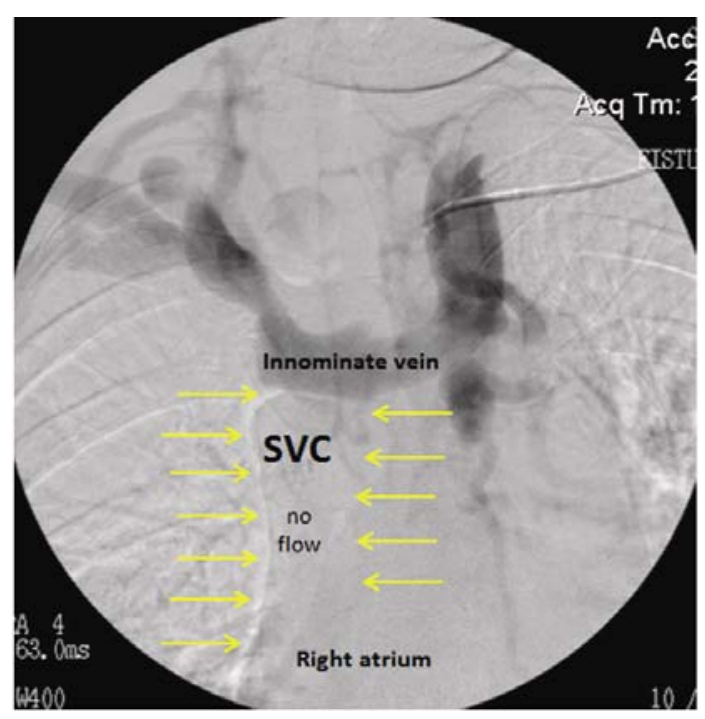

Figure 3. Occlusion of superior vena cava at junction with innominate vein. Arrows outlining the superior vena cava (SVC). 


\section{Discussion}

Acquired cutaneous lymphangiectasia is caused by lymph outflow obstruction, leading to increased lymphatic hydrostatic pressure and lymphedema. The lesions consist of groups of dilated, distorted, thin walled lymphatic channels in the superficial and mid dermis with very thin endothelium and varying numbers of smooth muscle cells ${ }^{[1,2]}$. An overlying hyperkeratotic or acanthotic epidermis is usually seen with these lesions ${ }^{[2]}$. Scattered lymphocytes and red blood cells can be present in the dilated lymphatic vessels, giving it a purple discoloration. Associated impaired immune function and fibrotic changes can follow lymphedema of the skin as a result of impairment of lymphatic function ${ }^{[2]}$. The skin lesions can be asymptomatic, pruritic, painful, burning, or present with chronic oozing or cellulitis ${ }^{[3]}$.

Determining the etiology of cutaneous lymphangiectasia and edema can be challenging, especially when confronted with only bland, nonspecific histology. In this case, SVC occlusion was raised based on the patient's history of chronic renal dialysis requiring multiple catheter placements and by increasing bilateral tender breast enlargement, "peau d'orange” skin changes and histology of lymphedema. We herein describe an unusual case in which the dermatopathologist made the initial awareness of SVC occlusion by seeing lymphangiectasia and stromal edema on a patient with bilateral breast enlargement.

Patients that are chronically hemodialyzed are more likely to develop SVC syndrome (SVCS), with a prevalence of $42 \%$ to $50 \%$ compared to $0.2 \%$ to $3.3 \%$ of patients with pacemakers ${ }^{[4]}$. There is some speculation that hemodialysis can result in alteration of clotting factors, platelet abnormalities, and increased levels of cytokines, C-reactive protein and homocysteine, inducing a pro-thrombotic state. Intravascular damage from increased venous pressures and from the placement of permanent intravenous catheters can also induce silent SVC stenosis. Chronic renal disease may also be an independent pre-thrombotic state, possibly predisposing patients to central vein occlusion. Diabetes mellitus is also a known risk factor ${ }^{[4]}$.

Other known causes of acquired cutaneous lymphangiectasia include: intralymphatic metastasis, lymphadenectomy, amputations, Crohn's disease, morphea profunda, systemic sclerosis, scrofuloderma, recurrent cellulitis, corticosteroid induced atrophy, cirrhotic ascites, porphyrias, and penicillamine induced dermopathy. There has been an increase in reported cases of acquired lymphangiectasia in the last 20 years, possibly secondary to an increase in radiation therapy for many cancers, excisional surgical procedures ${ }^{[5]}$, or increased awareness.

Symptoms of SVCS include headaches, dyspnea, chest pain, cough, face and neck swelling, upper extremity edema, dysphagia, and possibly minor bleeds. When the SVC obstruction occurs as a chronic process, it can instigate the development of subcutaneous collaterals which can expand to the mediastinum and abdominal or thoracic cavities ${ }^{[4]}$. This patient developed lymphangiectasia several months prior as a result of SVC obstruction, which had given an unusual presentation of bilateral breast enlargement and "peau d'orange" appearance. Acquired lymphangiectasia has been described to present clinically as clusters of thick-walled, translucent, fluid-filled papules ${ }^{[5]}$, but our patient instead presented with a clinical picture of mastitis. The patient's symptoms and clinical presentation subsided after treatment of the SVC occlusion.

The presence of end stage renal disease with an AV fistula for dialysis most likely contributed to the patient's venous stasis of the lateral cephalic outflow and SVC occlusion.

One of the venous collateral pathways recognized in SVC obstruction is the internal mammary pathway, which consists of the superficial veins of the thorax, internal mammary, and superior and inferior epigastric veins, which could explain the anatomical presentation of the lymphangiectasia in this case.

The differential diagnosis for stromal edema and dilated lymphatic vessels is broad, and may include other entities such as: intravascular histiocytosis, intralymphatic angioendothelioma, and reactive angioendotheliomatosis ${ }^{[5]}$. 
Treatment of lymphangiectasia consists of reduction of underlying lymphedema, and may consist of excision, surgery, carbon dioxide laser, electrocoagulation, cryotherapy, injection of a sclerosing agent, such as OK-432. Propranolol has also been used with some success in the treatment of congenital lymphangiomatosis, but has not yet been studied in acquired lymphangiectasia ${ }^{[3]}$.

\section{Conclusion}

We herein describe an unusual case in which a skin biopsy that only showed bland histological findings of stromal edema and lymphangiectasia led to a diagnosis of SVC occlusion. Clinical reports of similar presentations were not found. One should always consider the possibility of an underlying SVC occlusion when confronted with histological findings of lymphangiectasia, especially in those patients that are chronically hemodialyzed.

\section{References}

[1] Moon SE, Youn JI, Lee YS. Acquired cutaneous lymphangiectasia. British J of Dermatol. 1993; 129: $193-95$. http://dx.doi.org/10.1111/j.1365-2133.1993.tb03528.x

[2] Bolognia JL, Jorizzo JL, Schaeffer JV. Dermatology. 2012; 104: 1711-27.

[3] Bhat RM, Saldanha CS, Kambil SM, et al. Cutaneous lymohangiectasia of the vulva secondary to tuberculosis. In J Sex Transm Dis. 2012; 33(1): 35-37. PMid:22529452 http://dx.doi.org/10.4103/0253-7184.93817

[4] Pipili C, Cholongitas E, Tzanatos H. Two cases of silent superior vena cava synfrome associated with vascular access and end-stage renal disease. In J Artfic Org. 2009; 32(12): 883-8.

[5] Tomasini C, Butera AC, Pippione M. Acquired cutaneous lymphangiectasia with mesothelial cells reflux in a patient with cirrhotic ascites. Am J of Dermatopathol. 2008; 30(2): 140-44. PMid:18360117 http://dx.doi.org/10.1097/DAD.0b013e31816373ad

[6] Kistler AM, Silverman ED, Sharpe MB, et al. Superior vena cava obstruction in fibrosing mediastenitits: demonstration of right-to-left shunt and venous collaterals. Nuc Med Comm. 1991; 12: 1067-74.

http://dx.doi.org/10.1097/00006231-199112000-00008 